\title{
Correlation between initial spatial anisotropy and final momentum anisotropies in relativistic heavy ion collisions
}

\section{Sanchari Thakur, ${ }^{a, *}$ Sumit Kumar Saha, ${ }^{a}$ Pingal Dasgupta, ${ }^{b}$ Rupa Chatterjee ${ }^{a}$ and Subhasis Chattopadhyay ${ }^{a}$}

${ }^{a}$ Variable Energy Cyclotron Centre, HBNI, 1/AF, Bidhan Nagar, Kolkata-700064, India

${ }^{b}$ Key Laboratory of Nuclear Physics and Ion-beam Application(MOE),nstitute of Modern Physics, Fudan University, hanghai 200433, China

E-mail: s.thakur@vecc.gov.in, sk.saha@vecc.gov.in, pingaldg@fudan.edu.cn, rupa@vecc.gov.in, sub@vecc.gov.in

\begin{abstract}
The anisotropic flow $\left(v_{n}\right)$ in relaitvistic heavy ion collisions arises due to the initial spatial anisotropy $\left(\varepsilon_{n}\right)$ of the overlapping zone between the two colliding nuclei. The linear correlation between $\varepsilon_{n}$ and $v_{n}$ for small $\mathrm{n}$ is a quantitative measure of the efficiency of conversion of the initial spatial anisotropy to the final momentum anisotropy. We study the collision centrality, transverse momentum $\left(p_{T}\right)$ and collision energy dependence of this correlation for charged particles using a hydrodynamical model framework. The $p_{T}$ dependent correlation coefficient is found to be strongly dependent on the mass as well as $p_{T}$ of the emitted particles. We also study $p_{T}$ dependent relative fluctuation in anisotropic flow and show that it is sensitive to the value of $\eta / s$ specially in low $p_{T}$ region. In addition, we study the sensitivity of the correlation coefficient to the fluctuation size parameter.
\end{abstract}

The Ninth Annual Conference on Large Hadron Collider Physics - LHCP2021

7-12 June 2021

Online

${ }^{*}$ Speaker 


\section{Introduction}

The initial spatial asymmetry of the overlapping zone between two colliding nuclei gets converted into the final particles momentum anisotropy characterized by the flow coefficients which depend on particle mass, beam energy, collision centrality, transverse momentum etc. As the initial spatial anisotropy $\left(\varepsilon_{n}\right)$ increases from central to peripheral collisions, the flow coefficient $\left(v_{n}\right)$ also increases. The efficiency at which $\varepsilon_{n}$ is converted to $v_{n}$ depends on the initial state as well as on the evolution of the produced matter. Models based on relativistic hydrodynamics can be useful to estimate the initial states and the space time evolution of the matter which cannot be probed experimentally [1-11]. Several groups have studied the correlation between $\varepsilon_{n}$ and $v_{n}$ earlier $[1,10,12-26]$. It has been shown in different studies that the $p_{T}$, beam energy, collision centrality all play important role in the determination of the final momentum anisotropy [13, 27-31]. Thus, it is necessary to know the simultaneous effects of all these parameters on the correlation between $\varepsilon_{n}$ and $v_{n}$ better.

We study the $p_{T}$ dependent correlation between $\varepsilon_{n}$ and $v_{n}(\mathrm{n}=2 \& 3)$ using a hydrodynamical model for $\mathrm{Pb}+\mathrm{Pb}$ and $\mathrm{Cu}+\mathrm{Cu}$ collisions in this work [32]. We also study how the correlation coefficients and the relative fluctuations in the anisotropic flow parameters vary with the shear viscosity $(\eta / s)$ and fluctuation size parameter [33].

\section{Framework}

We use the $(2+1) \mathrm{D}$ longitudinally boost invariant hydrodynamical model framework MUSIC [32] with fluctuating initial conditions. The initial formation time of the plasma is taken as 0.4 $\mathrm{fm} / \mathrm{c}$. A Monte Carlo Glauber initial condition is used and the value of $\eta / s$ is chosen as 0.08 [33]. The fluctuation size parameter is taken as $0.4 \mathrm{fm}$. The freeze-out temperature is constant in our study and a lattice based equation of state is used for a cross-over transition between the QGP and hadronic matter phase [34]. The initial spatial eccentricity is calculated using the relation [13]:

$$
\epsilon_{n}=-\frac{\int \mathrm{d} x \mathrm{~d} y r^{n} \cos \left[n\left(\phi-\psi_{n}\right)\right] \varepsilon\left(x, y, \tau_{0}\right)}{\int \mathrm{d} x \mathrm{~d} y r^{n} \varepsilon\left(x, y, \tau_{0}\right)} .
$$

Where $\psi_{n}$ is the $n^{\text {th }}$ order participant plane angle. The corresponding anisotropic flow parameters $v_{n}$ can be obtained [13] from the invariant particle momentum distribution as :

$$
\frac{d N}{d^{2} p_{T} d Y}=\frac{1}{2 \pi} \frac{d N}{p_{T} d p_{T} d Y}\left[1+2 \sum_{n=1}^{\infty} v_{n}\left(p_{T}\right) \cos \mathrm{n}\left(\phi-\Psi_{\mathrm{n}}\right)\right]
$$

where $\Psi_{n}$ is $n^{\text {th }}$ order event plane angle.

The correlation coefficient $\mathrm{C}$ between the initial spatial eccentricity and final momentum anisotropies can be quantified using the relation [13]:

$$
C\left(\epsilon_{n}, v_{n}\right)=\left\langle\frac{\left(\epsilon_{n}-\left\langle\epsilon_{n}\right\rangle_{\mathrm{av}}\right)\left(v_{n}-\left\langle v_{n}\right\rangle_{\mathrm{av}}\right)}{\sigma_{\epsilon_{n}} \sigma_{v_{n}}}\right\rangle_{\mathrm{av}}
$$

Where, $\sigma_{\epsilon_{n}}$ and $\sigma_{v_{n}}$ are the standard deviations of $\epsilon_{n}$ and $v_{n}$ respectively. The average is taken over events using hadron multiplicity as weight factor. 


\section{Results of $\mathbf{P b}+\mathrm{Pb}$ collision}

It is well known that the mass ordering of differential anisotropic flow $\left(v_{n}\left(p_{T}\right)\right)$ parameters is a signature of the collective behaviour of the medium formed in heavy ion collisions and models based on hydrodynamics can explain it well. Therefore, it is necessary to know whether there exists any mass dependence in the $p_{T}$ dependent correlation coefficients for the different hadrons also. The correlation coefficient $\mathrm{C}\left(\epsilon_{n}, v_{n}\left(p_{T}\right)\right)$ for $\pi^{+}, K^{+}$and $p$ at $0-20 \%$ and $40-60 \%$ centralities is shown in Fig 1 [33]. It can be seen that there is a clear mass ordering in the correlation coefficient between $\epsilon_{2}-v_{2}$ for all the centrality bins.
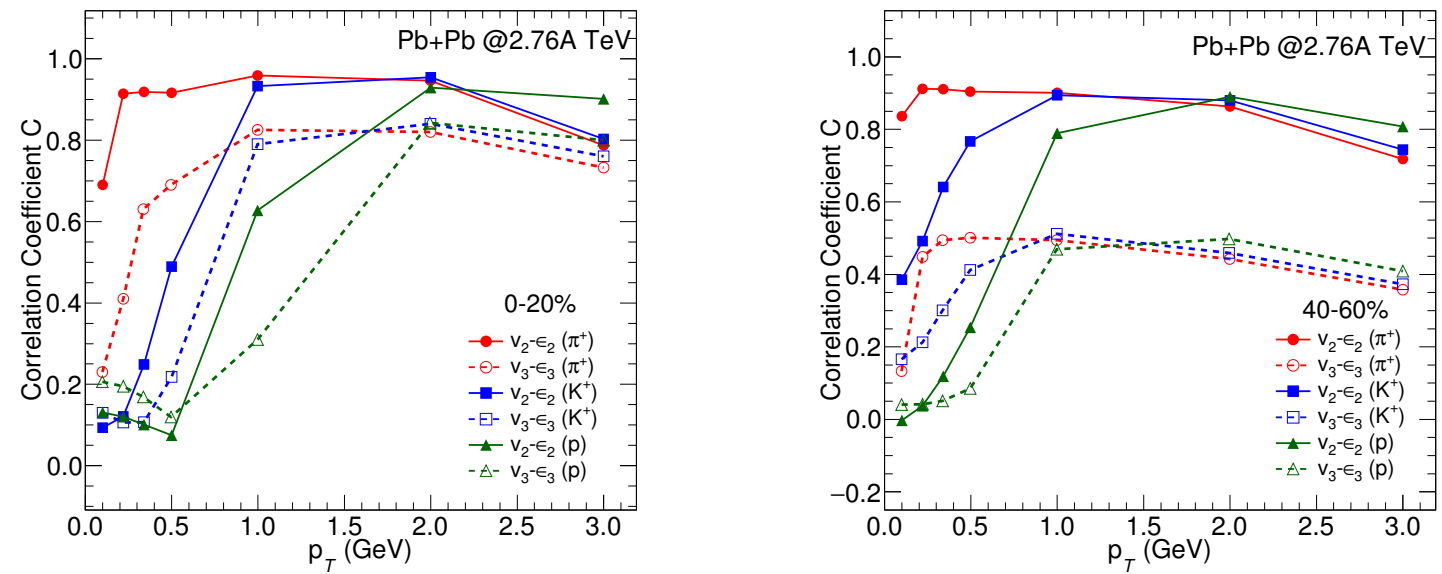

Figure 1: (Color online) $\mathrm{C}\left(\epsilon_{n}, v_{n}\left(p_{T}\right)\right)$ for $\pi^{+}, K^{+}$and $p$ at $\sqrt{s_{N N}}=2.76 \mathrm{TeV} \mathrm{Pb}+\mathrm{Pb}$ collisions for $0-20 \%$ and $40-60 \%$ centrality bins respectively [33].

However, there is no mass dependence in the $p_{T}$ integrated $\mathrm{C}\left(\epsilon_{n}-v_{n}\right)$ of $\pi^{+}, K^{+}$and $p$ with centralities [33] although the ( $p_{T}$ integrated) $v_{n}$ is different for them.

$\mathrm{C}\left(\epsilon_{3}, v_{3}\left(p_{T}\right)\right)$ has considerably smaller magnitude and it drops faster at higher $p_{T}$ values for peripheral collisions compared to central collision [33]. The results clearly show that the $p_{T}$ dependent correlation coefficient strongly depends on the mass of the particle and $p_{T}$ region that mainly contributes to the correlation strength is also different for different particles.

The relative fluctuation in the anisotropic flow parameters $\sigma_{v_{n}} /\left\langle v_{n}\right\rangle$, reflecting the ratio of the first two moments of the initial state eccentricity distribution (i.e, $\sigma_{\epsilon_{n}}$ ) is considered to be a potential observable. Fig 2 shows the sensitivity of the correlation coefficient and $\sigma_{v_{n}} /\left\langle v_{n}\right\rangle$ to the the value of the $\eta / s$. It can be seen that the value of $C$ for all the three particles varies only marginally, whereas, the $p_{T}$ dependent $\sigma_{v_{n}} /\left\langle v_{n}\right\rangle$ is quite sensitive to $\eta / s$ and the sensitivity is much stronger for protons than for pions and also in the low $p_{T}(<1 \mathrm{GeV})$ region for $\mathrm{Pb}+\mathrm{Pb}$ collisions [33].

\section{Results of $\mathrm{Cu}+\mathrm{Cu}$ collision}

The system produced in $\mathrm{Cu}+\mathrm{Cu}$ collisions at $200 \mathrm{~A} \mathrm{GeV}$ at $\mathrm{RHIC}$ is expected to have smaller temperature and energy density as well as smaller transverse dimension compared to $\mathrm{Pb}+\mathrm{Pb}$ collisions at $\mathrm{LHC}$. Also, the $\mathrm{Cu}+\mathrm{Cu}$ collision is expected to have a higher initial state density fluctuations. 

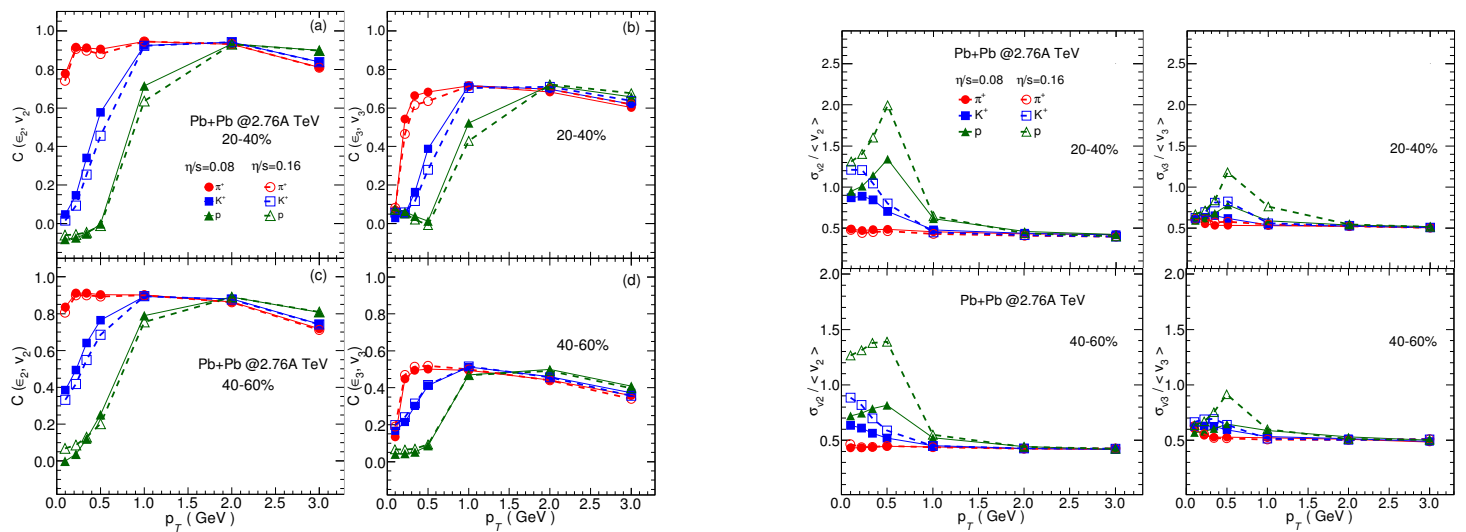

Figure 2: (Color online) [Left:] $p_{T}$ dependent correlation coefficients at the LHC considering two different $\eta / s$ values. [Right:] Relative fluctuations in the anisotropic flow parameters at the LHC considering two different $\eta / s$ values [33].
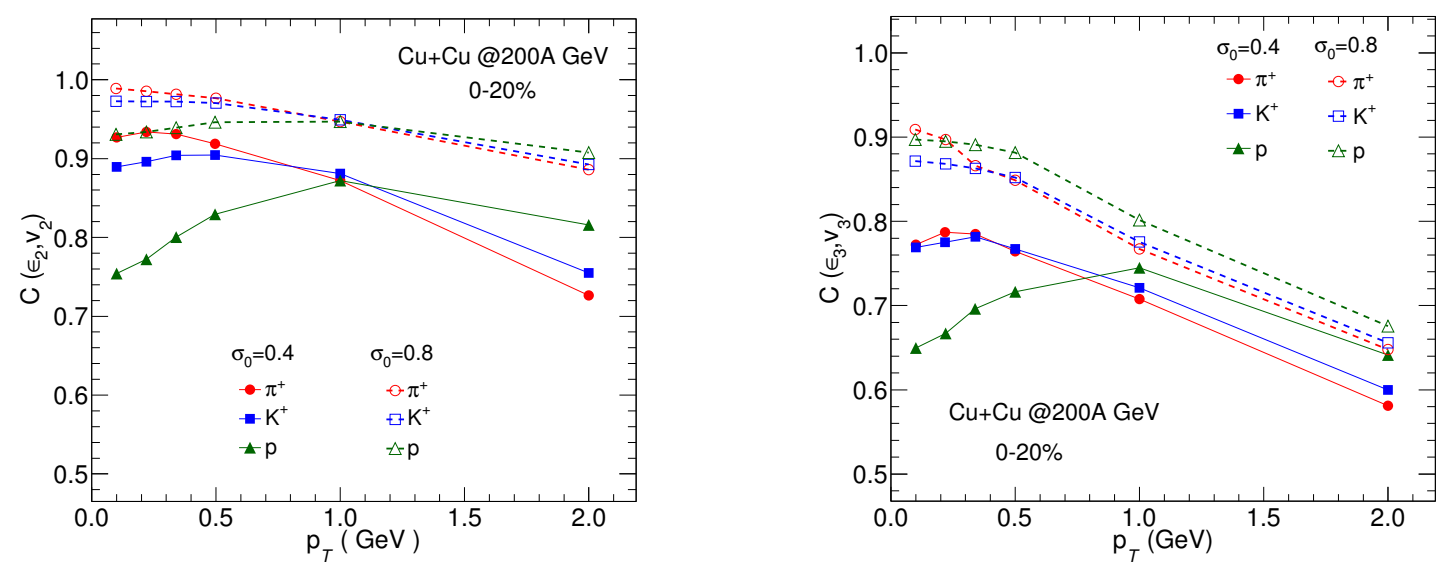

Figure 3: (Color online) $p_{T}$ dependent correlation coefficients for $\mathrm{Cu}+\mathrm{Cu}$ collision at $0-20 \%$ centrality for two fluctuation parameters.

A clear mass dependence of the correlation coefficient is seen in a relatively narrower $p_{T}$ range for $\mathrm{Cu}+\mathrm{Cu}$ collisions compared to $\mathrm{Pb}+\mathrm{Pb}$ collisions [33]. We also change the fluctuation size parameter $(\sigma)$ to see the sensitivity of correlation coefficient with $\sigma$ and the values of $\mathrm{C}$ for all the particles increase considerably with relatively smoother initial energy density distribution (for $\sigma=0.8 \mathrm{fm}$ ) [33] as shown in Fig. 3.

\section{Acknowledgment}

We like to thank the VECC grid computing facility and Abhisekh Seal for the continuous help throughout this work. PD acknowledges the support of the National Natural Science Foundation of China under Grants No. 11835002 and No. 11961131011. 


\section{References}

[1] H. Stoecker and W. Greiner, Phys. Rept. 137, 277-392 (1986).

[2] P. Kolb and U Heinz, Hydrodynamic description of ultrarelativistic heavy ion collisions Quark gluon plasma, R. C. Hwa (ed.) et al., p634 (2003).

[3] P. Huovinen, in Quark Gluon Plasma 3, edited by R. C. Hwa and X. N. Wang (World Scientific, Singapore, 2004), p. 600 [nucl-th/0305064];

[4] D. A. Teaney, arXiv:0905.2433 [nucl-th].

[5] P. F. Kolb, J. Sollfrank, and U. Heinz Phys. Rev. C 62, 054909 (2000).

[6] D. Teaney, J. Lauret, E.V. Shuryak, arXiv:0110037 [nucl-th].

[7] P. Huovinen and P. V. Ruuskanen, Ann. Rev. Nucl. Part. Sci. 56, 163 (2006).

[8] P. Romatschke and U. Romatschke, Phys. Rev. Lett. 99, 172301 (2007);

[9] J. Y. Ollitrault, Eur. J. Phys. 29, 275-302 (2008).

[10] A. Jaiswal and V. Roy, Adv. High Energy Phys. 2016, 9623034 (2016).

[11] L. Yan, Chin. Phys. C 42, 042001 (2018).

[12] G-Y. Qin, H. Petersen, S. A. Bass, and B. Muller, Phys. Rev. C 82, 064903 (2010).

[13] H. Niemi, G. S. Denicol, H. Holopainen and P. Huovinen, Phys. Rev. C 87, no.5, 054901 (2013).

[14] A.K. Chaudhuri, R. Haque, V. Roy, and B. Mohanty, Phys. Rev. C 87, 034907 (2013).

[15] F. G. Gardim, F. Grassi, M. Luzum, J.-Y. Ollitrault, Phys. Rev. C 85, 024908 (2012).

[16] A. K. Chaudhuri, Phys. Lett. B713, 91-92 (2012).

[17] L. Ma, G. L. Ma, and Y. G. Ma, arXiv:0610:04733

[18] A. Bzdak, B. Schenke, P. Tribedy, and R. Venugopalan, phys. Rev. C 87, 064906 (2013).

[19] V. Greco, M.Colonna, M. Di Toro, G.Ferini Prog. in part. and Nucl. Phys 62, 562, 2009.

[20] B. H. Alver, C. Gombeaud, M. Luzum and J. Y. Ollitrault, Phys. Rev. C 82, 034913(2010).

[21] J. Noronha-Hostler, L. Yan, F. G. Gardim, and J.-Y. Ollitrault, Phys. Rev. C 93, (2016).

[22] P. Alba, V. Mantovani Sarti, J. Noronha, J. Noronha-Hostler, P. Parotto, I. Portillo Vazquez, and C. Ratti, Phys. Rev. C 98, 034909 (2018).

[23] M. Sievert and J. Noronha-Hostler, Phys. Rev. C 100, 024904 (2019). 
[24] R. Snyder, M. Byres, S.H. Lim, J.L. Nagle, arXiv:2008:08729

[25] M. Luzum, C. Gombeaud, and J.-Y. Ollitrault, Phys. Rev. C 81, 054910 (2010).

[26] F. G. Gardim, F. Grassi, M. Luzum, and J. Noronha-Hostler, Phys. Rev. C 95, 034901 (2017).

[27] M. Alvioli, H. Holopainen, K. J. Eskola, and M. Strikman, Phys. Rev. C 85, 034902 (2012).

[28] G. Aad et. al., Eur. Phys. J C 79, 985 (2019).

[29] P. Bozek, Phys. Rev. C 93, 044908 (2016).

[30] P. Bozek and H. Mehrabpour, Phys. Rev. C 101, 064902 (2020).

[31] G. Giacalone, F. G. Gardim, J. Noronha-Hostler and J. Y. Ollitrault, Phys. Rev. C 103 no.2, 024909 (2021).

[32] B. Schenke, S. Jeon, and C. Gale, Phys. Rev. C 82, 014903 (2010).

[33] S. Thakur, S. K. Saha, P. Dasgupta, R. Chatterjee and S. Chattopadhyay, Nucl. Phys. A 1014 (2021), 122263 doi:10.1016/j.nuclphysa.2021.122263 [arXiv:2101.09998 [nucl-th]].

[34] P. Huovinen and P. Petreczky, Nucl. Phys. A 837, 26-53 (2010). 